Pacific Journal of Mathematics

REPRESENTATIONS OF THE MAUTNER GROUP. 


\title{
REPRESENTATIONS OF THE MAUTNER GROUP, I
}

\section{LARRY BAGGETT}

\begin{abstract}
The five-dimensional connected Lie group known as the "Mautner group" is not of type $I$. In fact all of its irreducible unitary representations are not yet known. We present here a discussion of the known representations and produce a five-parameter family of new representations.
\end{abstract}

I. Introduction. By the Mautner group we shall mean the semidirect product $G=C^{2} R$ of two-dimensional complex space $C^{2}$ with the real line $R$ in which multiplication is given by $\left((z, w, t)\left(z^{\prime}, w^{\prime}, t^{\prime}\right)\right)=$ $\left(z+e^{i t} z^{\prime}, w+e^{2 \pi i t} w^{\prime}, t+t^{\prime}\right)$. This group is distinguished by being the "Smallest" connected Lie group whose unitary dual, space of equivalence classes of irreducible unitary representations, remains unknown. In particular, Mackey's theory for semi-direct products is insufficient in this case, the Mautner group not being a "Regular" semi-direct product. Our goal has been to develop new techniques for constructing irreducible representations of $G$ which would supplement Mackey's method and hopefully provide a realization of all the irreducible unitary representations of the Mautner group together with a reasonable criterion for their mutual equivalence. This group is not of type I, (indeed Mautner himself discovered it as the first example of a connected Lie group which was not of type I), so that its unitary dual is not "Smooth." We will then not be able to catalogue the entire dual by any "Nice" space of parameters, but we can hope that an adequate description of the dual, if less precise, does exist. For example, in this first paper we shall produce a series $\left[U^{(r, s, \lambda, \mu, d)}\right]$ of irreducible representations of $G$, with $r$ and $s$ positive real numbers, $\lambda$ and $\mu$ real numbers, and $d$ an integer, which satisfies:

THEOREM 1.1. $U^{(r, s, \lambda, \mu, d)}$ is equivalent to $U^{\left(r^{\prime}, s^{\prime}, \lambda^{\prime}, \mu^{\prime}, d^{\prime}\right)}$ if and only if $r=r^{\prime}, s=s^{\prime}, d=d^{\prime}$, and $\lambda+d \mu=\lambda^{\prime}+d^{\prime} \mu^{\prime}+p+2 \pi q$, for some integers $p$ and $q$. For $d$ unequal to zero, the representations $\left[U^{(r, s, \lambda, \mu, d)}\right]$ are "New" representations of $G$.

We mention here that the theory of representations induced from virtual subgroups of $G$, invented by Mackey and developed by Ramsay in [5], classifies completely, in a sense, the entire unitary dual. Really it only replaces the problem of constructing the irreducible unitary representations of $G$ by the two problems: (a) finding all the virtual subgroups of $G$, (very difficult and not yet solved even in this simplest case), and (b) finding all the irreducible representations of these 
virtual subgroups, (harder yet). Our idea is to circumvent these problems by discovering methods, alternative to inducing, for constructing irreducible representations.

To make clear what we shall mean by a "New" representation of the Mautner group, let us recall where Mackey's procedure leaves us. The Mautner group acts, by innter automorphisms, on the normal subgroup $C^{2}$ of $G$, and therefore it acts dually on the dual group $\left[C^{2}\right]^{\wedge}$ of $C^{2}$ the character $(\chi, \varphi)$ of $C^{2}$ being transformed by the group element $(z, w, t)$ to the character $\left(e^{-i t} \chi, e^{-2 \pi i t} \varphi\right)$. We let $S_{r}$ denote the circle in the complex plane with center at the origin and radius $r \geqq 0$.

THEOREM 1.2. (Mackey) Let $\pi$ be an irreducible representation of $G$. Let $\mathscr{P}^{\pi}$ be the projection-valued measure on $\left[C^{2}\right]^{\wedge}$ corresponding, via Stone's theorem, to the representation $\left.\pi\right|_{\left[C^{2}\right]}$. Then $\mathscr{P}^{\pi}$ is concentrated on a unique torus $S_{r} \times S_{s}$ in $\left[C^{2}\right]^{\wedge}$. Further, $\mathscr{P}^{\pi}$ is completely determined by a "Quasi-Invariant" measure $\Sigma$ on $S_{r} \times S_{s}$ and an integer $j$. (A measure $\Sigma$ is called "Quasi-Invariant" with respect to an action of a group $G$ if $\Sigma(E \cdot g)=0$ whenever $\Sigma(E)=0$.)

A "Mackey" representation of $G$ is a representation $\pi$ such that $\mathscr{P}^{\pi}$ is concentrated on some orbit in $\left[C^{2}\right]^{\wedge}$. This happens if and only if $\mathscr{P}^{\pi}$ gives nonzero measure to some orbit. It $\pi$ is not a Mackey representation, then we say that $\pi$ is associated with the quasi-orbit $\Sigma$ and that it has multiplicity $j$.

The Mackey representations are exactly the ones obtained by his procedure. In many cases these Mackey representations exhaust the entire set of irreducible representations. However, in the case of the Mautner group we may easily write down an irreducible nonMackey representation. Thus let $r$ and $s$ be posititive real numbers, and define a representation $\pi^{(r, s)}$ of $G$, acting in $L^{2}\left(S_{1} \times S_{1}\right)$, and given by

$$
\left(\pi_{(z, w, t)}^{(r, s)} f\right)(\alpha, \beta)=e^{i(r \alpha, z)} e^{i(s \beta, w)} f\left(\alpha e^{-i t}, \beta e^{-2 \pi i t}\right),
$$

where $($,$) denotes the real vector space inner product in the complex$ plane $C$.

One may verify directly that $\pi^{(r, s)}$ is irreducible, that it is associated with the Lebesgue measure quasi-orbit of $S_{r} \times S_{s}$, and that it has multiplicity 1.

There is a slight generalization of these representations. Recall that if $\pi$ is an irreducible representation of $G$ and if $\gamma$ is a character of $G$, then $\gamma \pi$ also is an irreducible representation. The characters of the Mautner group are the mappins $(z, w, t) \Rightarrow e^{i \lambda t}$ for $\lambda$ real. Define then representations $\left[\pi^{(r, s, \lambda)}\right]$ of $G$ by $\pi_{(z, w, t)}^{(r, s, \lambda)}=e^{i \lambda t} \pi_{(z, w, t)}^{(r, s)}$. 
THEOREM 1.3. Each $\pi^{(r, s, \lambda)}$ is irreducible. We have that $\pi^{(r, s, \lambda)}$ is equivalent to $\pi^{\left(r^{\prime}, s^{\prime}, \lambda^{\prime}\right)}$ if and only if $r=r^{\prime}, s=s^{\prime}$, and $\lambda=\lambda^{\prime}+$ $p+2 \pi q$ for some integers $p$ and $q$.

Proof. The irreducibility has already been observed. If $r=r^{\prime}$, $s=s^{\prime}$, and $\lambda=\lambda^{\prime}+p+2 \pi q$ for some integers $p$ and $q$, then the operator $A$ on $L^{2}\left(S_{1} \times S_{1}\right)$ defined by $A f(\alpha, \beta)=\alpha^{p} \beta^{q} f(\alpha, \beta)$ effects the equivalence between $\pi^{(r, s, \lambda)}$ and $\pi^{\left(r^{\prime} s^{\prime} \lambda^{\prime}\right)}$.

Finally, suppose that $\pi^{(r, s, \lambda)}$ is equivalent to $\pi^{\left(r^{\prime} s^{\prime} \lambda^{\prime}\right)}$. First consider their restrictions to the subgroup $C^{2}$. The one is concentrated (by inspection) on the torus $S_{r} \times S_{s}$, while the other is concentrated on the torus $S_{r^{\prime}} \times S_{s^{\prime}}$. By the uniqueness of that torus we have that $r=r^{\prime}$ and $s=s^{\prime}$. Next consider their restrictions to the subgroup $R$. We have

$$
\left(\pi_{(0,0, t)}^{(r, s)} f\right)(\alpha, \beta)=e^{i \lambda t} f\left(\alpha e^{-i t}, \beta e^{-2 \pi i t}\right),
$$

while

$$
\left(\pi_{(0,0, t)}^{\left(r, s, \ell^{\prime}\right)} f\right)(f)(\alpha, \beta)=e^{i \lambda^{\prime} t} f\left(\alpha e^{-i t}, \beta e^{-2 \pi i t}\right) .
$$

The first representation decomposes into a direct sum of the characters $t \rightarrow e^{i t(\lambda+p+2 \pi q)}$ for $p$ and $q$ running over the set of integers. (The functions $\alpha^{p} \beta^{q}$ form an orthonormal basis of eigenvectors.) On the other hand the second representation decomposes as a direct sum of the characters $t \Rightarrow e^{i t\left(\lambda^{\prime}+p+2 \pi q\right)}$ for $p$ and $q$ running over the set of integers. Clearly, in order for these representations to be equivalent, we must have $\lambda^{\prime}=\lambda+p+2 \pi q$ for some integers $p$ and $q$.

These representations $\left[\pi^{(r, s, \lambda)}\right]$ certainly have been known to researchers for some time. Similar constructions can be made beginning with any quasi-invariant measure $\Sigma$ on the torus $S_{1} \times S_{1}$, and a similar series of representations can be produced. Such measures are not well understood, although their existence in abundance is assured. One of the aspirations of the author is to discover one of these "Other" representation through a different method of construction. In any event, to the author's knowledge the representations $\left[\pi^{(r, s, \lambda)}\right]$ are the only known examples of irreducible unitary representations of the Mautner group which are associated with the Lebesgue measure quasi-orbit on the torus $S_{r} \times S_{s}$. In $\S I V$ we shall produce an enlarged list of such representations. All the known representations, as well as those presented in this article, have multiplicity 1 . In the next paper we shall produce irreducible representations associated with the Lebesgue measure quasi-orbit but having multiplicity $j>1$. 
Our method of constructing "New" representations of the Mautner group begins as follows: take $N$ to be the closed normal subgroup of $G$ consisting of the triples $(0, w, 0)$. Then $N$ is regularly imbedded in $G$, (all the orbits in $\hat{N}$ are circles), and we may apply the Mackey machine, reducing the problem of determining the unitary dual of $G$ to the problem of determining the duals of the various "Little Groups" which occur. But for any nonzero character $\varphi$ of $N$, the stability subgroup $H$ of $G$ for $\varphi$ consists of the triples $(z, w, n), n$ an integer. So only one nontrivial little group occurs and it is isomorphic with the "Discrete Mautner Group" $D$ which is defined to be the semi-direct product $C Z$ of the complex plane $C$ with the group $Z$ of integers, where multiplication is given by $\left((z, n)\left(z^{\prime}, n^{\prime}\right)\right)=$ $\left(z+e^{i n} z^{\prime}, n+n^{\prime}\right)$.

THEOREM 1.4. (Mackey) Let $V$ be an irreducible representation of the discrete Mautner group $D$. Define a representation $S$ of the stability subgroup $H$ of $G$ for the nonzero character $\varphi$ of $N$ by $S_{(z, w, n)}=e^{i(\varphi, w)} S_{(z, n)}$, where as before (, ) denotes the real inner product in $C$. Define a representation $U$ of $G$ by $U=\operatorname{ind}_{H}^{G} S$. Then the mapping $V \Longrightarrow U$ is a one-to-one, equivalence-preserving, correspondence between the set of all irreducible representations of $D$ and the set of all irreducible representations $U$ of $G$ which are associated with the orbit of $\varphi$ in $\hat{n}$.

This marvelous theorem of Mackey enables us to replace the problem of determining the unitary dual of $G$ by the "Presumably easier" problem of determining the unitary dual of the discrete Mautner group $D$. Of course $D$ also is not a regular semi-direct product. Indeed $D$ must be as complicated as is $G$. If $V$ is a Mackey representation of $D$, then the corresponding $U$ is a Mackey representation of $G$. There are nonMackey representations of $D$. Thus if $r$ is a positive real number and $\lambda$ is real, define a representation $\rho^{(r, \lambda)}$ of $D$, acting in $L^{2}\left(S_{1}\right)$, and given by

$$
\left(\rho_{(z, n)}^{(r, \lambda)} f\right)(\alpha)=e^{i \lambda n} e^{i(r \alpha, z)} f\left(\alpha e^{-i n}\right) .
$$

THEOREM 1.5. Each $\rho^{(r, \lambda)}$ is irreducible and is not a Mackey representation of $D$. We have that $\rho^{(r, \lambda)}$ is equivalent to $\rho^{\left(r^{\prime}, \lambda^{\prime}\right)}$ if and only if $r=r^{\prime}$ and $\lambda=\lambda^{\prime}+p+2 \pi q$ for some integers $p$ and $q$.

Proof. This proof is completely analogous to the proof of Theorem 1.3. We omit the details. 
Now if $V$ is the representation $\rho^{(r, 2)}$ of $D$, and if $s=\|\phi\|$, then the representation $U$ of $G$ corresponding to $V$ as in Theorem 1.4 is the representation $\pi^{(r, s, \lambda)}$. Hence the "Known" representation of $D$ lead, a la 1.4, to the "Known" representations of $G$. So what we shall in fact do here is to construct "New" representations of $D$.

REMARK. In $\S I V$ we shall explicitly calculate the mapping $V \Rightarrow U$ of Theorem 1.4. The two statements just made about that mapping will then be clear.

In $\S I I$ we introduce the idea of the "Generalized Tensor Product" of representations. This is in a sense the only new notion in this paper. We apply this method in §III to construct the new representations of $D$, and then in $\S I V$ we construct the new representations of $G$. Even though we are endeavoring to circumvent the Mackey machine, it is always lurking in the background. The reader who has some familiarity with those ideas will be well-prepared to follow these arguments.

Many of the results contained here were presented in an informal seminar at the conference on representations of Lie groups held at Oxford during July of 1977. The author would like to express his appreciation to the organizers and participants for giving him the opportunity to speak there. He also would like to thank Professor Arlan Ramsay for much valuable counsel.

II. Generalized tensor products of representations. Let $G$ denote an arbitrary locally compact group. One way of constructing irreducible representations of $G$, given at least one nontrivial representation to start with, is by taking tensor products and then decomposing into irreducible constituents. For example we have the so-called "Stone-Weierstrass" theorem for compact groups.

Theorem 2.1. (Disguised Peter-Weyl theorem) Let G be a compact group, and let $\mathscr{T}$ be a collection of representations of $G$ which separates the points of $G$. Then every irreducible representation of $G$ is a subrepresentation of some tensor product of finitely many representations which either are elements of $\mathscr{T}$ or are conjugate to elements of $\mathscr{T}$.

Although no such result holds for noncompact groups, one might expect to be able to construct some new representations from old ones via this method. For example, in the case we are pursuing $G$ is the discrete Mautner group $D$, and we do have some representations to begin with, namely the Mackey representations and the $\left[\rho^{(r, \lambda)}\right]$. However, what happens is the following: 
THEOREM 2.2. The tensor product of two Mackey representations of $D$ is a direct sum of other Mackey representions. The tensor product of a $\rho^{(r, \lambda)}$ with a $\rho^{\left(r^{\prime}, \lambda^{\prime}\right)}$ is a direct integral of other $\rho^{\left(r^{\prime \prime}, \lambda^{\prime \prime}\right)}$ s. The tensor product of a Mackey representation with $a \rho^{(r, \lambda)}$ is a direct sum of other $\rho^{\left(r^{\prime}, \lambda^{\prime}\right)}$ s.

These facts follow more or less directly from Mackey's analysis. Since the results are negative from our point of view, we shall not even include the proof.

A procedure which does yield new representations is one which we shall call a "Generalized Tensor Product." Let us think of the ordinary tensor product of two representation $V$ and $W$ of $G$ as the restriction to the diagonal subgroup of $G \times G$ of the outer Kronecker product $V \times W$ of $V$ and $W$. We shall generalize the notion of this outer product.

The ordinary outer Kronecker product of $V$ and $W$ is defined as follows. It acts in the tensor product of the Hilbert spaces $X(V)$ and $X(W)$, and it is given by the formula $(V \times w)_{\left(g_{1}, g_{2}\right)}=V_{g_{1}} \otimes W_{g_{2}}$. If we try to describe this process entirely within the group $G \times G$, we may formulate it in a slightly different manner. Let $V^{*}$ be the representation of the subgroup $G \times[e]$ defined by $V_{(g, e)}^{*}=V_{g}$, and let $* W$ be the representation of the subgroup $[e] \times G$ defined by $* W_{(e, g)}=$ $W_{g}$. We then see that $(V \times W)_{\left(g_{1}, g_{2}\right)}=V_{\left(g_{1}, e\right)}^{*} \otimes{ }^{*} W_{\left(e, g_{2}\right)}$, which we can interpret as $V_{\left(g_{1}, g_{2}\right)}^{* \prime} \otimes{ }^{*} W_{\left(g_{1}, g_{2}\right)}^{\prime}$, where $V^{* \prime}$ and ${ }^{*} W^{\prime}$ are the obvious extensions of $V^{*}$ and ${ }^{*} W$ to all of $G \times G$. The point is that there are other extensions of $V^{*}$ and ${ }^{*} W$ to all of $G \times G$, and we make use of these others to form generalized outer Kronecker products and generalized tensor products.

DEFINITION 2.3. Let $V$ and $W$ be representations of a locally compact group $G$. Let $V^{*}$ and ${ }^{*} W$ be the representations defined on the subgroups $G \times[e]$ and $[e] \times G$ as defined in the above paragraph. Let $V^{* 1}$ and ${ }^{*} W^{1}$ be extensions of $V^{*}$ and ${ }^{*} W$ to all of $G \times G$. Then the Generalized Outer Kronecker Product of $V$ and $W$ Depending on the Extensions $V^{* 1}$ and ${ }^{*} W^{1}$ is the representation of $G \times G$, acting in the tensor product of the spaces $X(V)$ and $X(W)$, and given by the mapping $\left(g_{1}, g_{2}\right) \Rightarrow V_{\left(g_{1}, g_{2}\right)}^{* 1} \otimes * W_{\left(g_{1}, g_{2}\right)}^{1}$. The Generalized Tensor Product of $V$ and $W$ Depending on the Extensions $V^{* 1}$ and ${ }^{*} W^{1}$ is the restriction to the diagonal subgroup of the outer product.

REMARK 1. If $V^{* 1}$ and ${ }^{*} W^{1}$ are the obvious extensions of $V^{*}$ and ${ }^{*} W$, then the generalized products agree with the ordinary ones. If there is to be any value to this idea, it will come from the existence of other extensions. 
REMARK 2. Although the obvious extensions of $V^{*}$ and ${ }^{*} W$ are unitary representations of $G \times G$, the definition makes perfectly good sense if we allow multiplier representations. Indeed it is this situation which can produce new representations of $G$. Often the multipliers for $V^{* 1}$ and ${ }^{*} W^{1}$ will cancel each other out anyway.

REMARK 3. If $V^{* 1}$ and $V^{* 2}$ are cohomologous extensions of $V^{*}$, i.e., if $V^{* 2}=\tau V^{* 1}$ where $\tau$ is a scalar function, and if ${ }^{*} W^{1}$ and ${ }^{*} W^{2}$ are cohomologous extensions of ${ }^{*} W$, say ${ }^{*} W^{2}=\Sigma^{*} W^{1}$, then the two generalized tensor products of $V$ and $W$ depending on the extensions $V^{* 1}$ and ${ }^{*} W^{1}$ and on the extension $V^{* 2}$ and ${ }^{*} W^{2}$ will be cohomologous representations of $G$. Indeed they will differ by the scalar function $g \Longrightarrow \tau(g, g) \Sigma(g, g)$. So if the resulting tensor products are unitary representations of $G$, they will only differ by a character. In other words, in order to construct new representations of $G$, we need only consider one extension $V^{* 1}$ from each cohomology class of extensions of $V^{*}$. Hence, if there is to be any metrit to this idea, there must be some noncohomologous extensions of $V^{*}$ and $* W$.

REMARK 4. In [1] the set of all cohomology classes of extensions of a representation $V^{*}$ to all of $G \times G$ is completely determined. They are classified by a group of homomorphisms of $G$ into a certain abelian group $\Gamma\left(V^{*}\right)$. Sometimes this abelian group $\Gamma\left(V^{*}\right)$ is trivial, in which case the generalized tensor products give nothing new. Sometimes the group hom $\left(G, \Gamma\left(V^{*}\right)\right)$ is trivial, and again nothing new is forthcoming. In fact, if we try this procedure for the connect Mautner group $G$ and for one of the representations $\pi^{(r, s, \lambda)}$, we find that indeed $\Gamma\left(V^{*}\right)$ is nontrivial but hom $\left(G, \Gamma\left(V^{*}\right)\right)$ is trivial.

The point is that for the discrete Mautner group there do exist nontrivial extensions and new representations do occur. In the next section we shall recall the construction procedure for these other extensions. We then apply the generalized tensor product method to construct new representations of $D$.

III. Some new representations of the discrete Mautner group. As before we let $D$ be the semi-direct product $C Z$ of the complex $C$ with the group $Z$ of integers where multiplication is given by $(z, n)\left(z^{\prime}, n^{\prime}\right)=\left(z+e^{i n} z^{\prime}, n+n^{\prime}\right)$. Let $r$ and $r^{\prime}$ be positive numbers and let $\lambda$ and $\lambda^{\prime}$ be real numbers. Let $V$ and $W$ be the representations $\rho^{(r, \lambda)}$ and $\rho^{\left(r^{\prime}, \lambda^{\prime}\right)}$ respectively. (See $\S I$ for the definitions of these representations of $D$.) We wish to compute some generalized tensor products of $V$ and $W$.

To begin with we let $V^{*}$ be the representation of $D \times[e]$ defined by $V_{(d, e)}^{*}=V_{d}$. In order to construct the different extensions of $V^{*}$ 
to all of $D \times D$, we must first, according to [1], determine the group $\Gamma\left(V^{*}\right)$ of all characters $\gamma$ of $D \times[e]$ for which $\gamma V^{*}$ is equivalent to $V^{*}$. Now the characters of $D \times[e]$ are in one-to-one correspondence with the elements of the unit circle, the element $\psi$ determining the character $((z, n), e) \Rightarrow \psi^{n}$.

Proposition 3.1. $\Gamma\left(V^{*}\right)$ consists of the elements \& of the unit circle of the form $\psi=e^{i p}$ for $p$ an integer.

Proof. First let $\psi=e^{i p}$, and define an operator $U_{\psi}$ on $L^{2}\left(S_{1}\right)$, the space of $V^{*}$, by $U_{\psi} f(\alpha)=\alpha^{p} f(\alpha)$. Then we have that

$$
\begin{aligned}
\left(U_{\psi} V_{(z, n), e)}^{*} U_{\bar{\psi}^{1}}^{-1} f\right)(\alpha) & =\alpha^{p}\left(V_{(z, n), e)}^{*} U_{\psi^{-1}}^{-1} f\right)(\alpha) \\
& =\alpha^{p}\left(V_{(z, n)} U_{\bar{\psi}^{1}}^{-1} f\right)(\alpha)=\alpha^{n}\left(\rho_{(z, n)}^{(r, \lambda)} U_{\bar{\psi}^{-1}}^{-1} f\right)(\alpha) \\
& =\alpha^{p} e^{i \lambda n} e^{i(r \alpha, z)}\left(U_{\bar{\psi}^{1}}^{-1} f\right)\left(\alpha e^{-i n}\right) \\
& =\alpha^{p} e^{i \lambda n} e^{i(r \alpha, z)} \alpha^{-p} e^{i n p} f\left(\alpha e^{-i n}\right) \\
& =\psi^{n}\left(\rho_{(z, n)}^{(r, \lambda)} f\right)(\alpha)=\psi^{n}\left(V_{(z(n), e)}^{*} f\right)(\alpha),
\end{aligned}
$$

which shows that $\psi$ belongs to $\Gamma\left(V^{*}\right)$.

To see that this exhausts $\Gamma\left(V^{*}\right)$, let $\psi$ be a character of $D \times[e]$ and consider the restrictions of the representations $V^{*}$ and $\psi V^{*}$ to the subgroup $Z \times[e]$ of $D \times[e]$. We have that

$$
\left(V_{(z, n), e)}^{*} f(\alpha)=e^{i \lambda n} f\left(\alpha e^{-i n}\right),\right.
$$

and

$$
\left(\left(\psi V^{*}\right)_{((z, n), e)} f\right)(\alpha)=\psi^{n} e^{i \lambda n} f\left(\alpha e^{-i n}\right)
$$

The first of these representations of $Z$ decomposes into a direct sum of the characters $n \rightarrow e^{i n(\lambda+p)}$ for $p$ running through the integers, while the second representation decomposes into the direct sum of the characters $n \Rightarrow e^{i n(\lambda+p)} \psi^{n}$. The only way for these two representations to be equivalent is for $\psi$ to be $e^{i p}$ for some $p$.

REMARK 1. Notice that the group $\Gamma\left(V^{*}\right)$ does not depend on the parameters $r$ and $\lambda$. Note also that the same kinds of arguments would show that $\Gamma\left(^{*} W\right)$ would consist of exactly the same elements $\left[e^{i p}\right]$ of the circle.

REMARK 2. The construction of all the extensions of $V^{*}$ to $D \times D$ requires that we know a mapping $\psi \Rightarrow U_{\psi}$ of $\Gamma\left(V^{*}\right)$ into the unitary group on $X\left(V^{*}\right)$ such that $U_{\psi} V_{(z, n), e)}^{*} U_{\bar{\psi}^{1}}{ }^{2} \psi^{n} V_{((z, n), e)}^{*}$ (See [1].) As a matter of fact we have seen in the course of the last proof what this mapping is. Thus 


$$
\left(U_{\psi} f\right)(\alpha)=\alpha^{p} f(\alpha) \text { where } \psi=e^{i p} .
$$

Next we must compute the group hom $\left(D, \Gamma\left(V^{*}\right)\right)$, which is the group hom $(D, Z)$, which consists of the mappings $(w, k) \Rightarrow j k$ for some integer $j$. In other words, if $h$ is an element of hom $\left(D, \Gamma\left(V^{*}\right)\right)$, then there exists an integer $j$ such that the character $h(w, k)$ sends the element $((z, n), e)$ of $D \times[e]$ to the number $e^{i j k n}$.

Proposition 3.2. In this case the group $\mathscr{F}\left(V^{*}\right)$, see [1], is isomorphic to the group of integers.

REMARK. Exactly similar calculations and results hold for the group $\mathscr{F}\left({ }^{*} W\right)$.

We are now ready to write down typical extensions of $V^{*}$ and ${ }^{*} W$. Fix integers $j$ and $j^{\prime}$. We shall construct the generalized tensor product of $V$ and $W$ depending on the extensions $V^{* j}$ and ${ }^{*} W^{j^{\prime}}$ determined, as in [1], by the homomorphisms in $\mathscr{F}\left(V^{*}\right)$ and $\mathscr{F}\left({ }^{*} W\right)$ corresponding respectively to the integers $j$ and $j^{\prime}$. The Mackey extension of $V^{*}$ is the obvious one. We have then that

$$
\left(V_{((z, n),(w, k))}^{* j} f\right)(\alpha)=\left(V_{(z, n)} U_{\left(h^{j}(w, k)\right)} f\right)(\alpha),
$$

(where $h^{j}$ is the homomorphism corresponding to $j$ ),

$$
\begin{aligned}
& =\left(\rho_{(z, n)}^{(r, \lambda)} U_{\left(e^{i j k)}\right)} f\right)(\alpha) \\
& =e^{i \lambda n} e^{i(r \alpha, z)}\left(U_{\left(e^{i j k)}\right)} f\right)\left(\alpha e^{-i n}\right) \\
& =e^{i \lambda n} e^{i(r \alpha, z)} \alpha^{j k} e^{-i j k n} f\left(\alpha e^{-i n}\right) .
\end{aligned}
$$

Similarly we obtain the following extension ${ }^{*} W^{j^{\prime}}$ of ${ }^{*} W$.

$$
\begin{aligned}
& \left({ }^{*} W_{((z, n),(w, k))}^{j^{\prime}} f\right)(\beta) \\
& \quad=e^{i \lambda^{\prime} k} e^{i\left(r^{\prime} \beta, w\right)} \beta^{j^{\prime} n} e^{-i j^{\prime} k n} f\left(\beta e^{-i k}\right) .
\end{aligned}
$$

The generalized tensor product of $V$ and $W$ depending on these extensions is then a representation $V^{\left(r, r^{\prime}, j, j^{\prime}\right)}$, acting in $L^{2}\left(S_{1} \times S_{1}\right)$, and given by

$$
\begin{aligned}
& \left(V_{(z, n)}^{\left(r, r^{\prime}, j, j^{\prime}\right)} f\right)(\alpha, \beta) \\
& \quad=e^{i n\left(\lambda+\lambda^{\prime}\right)} e^{i\left(\left(r \alpha+r^{\prime} \beta\right), z\right)} \alpha^{j n} \beta^{j^{\prime} n} e^{-i n^{2}\left(j+j^{\prime}\right)} f\left(\alpha e^{-i n}, \beta e^{-i n}\right) .
\end{aligned}
$$

We observe that this representation is a direct integral over the unit circle, (think of $\beta=\alpha \theta$ ), of representations $S^{\left(r, r^{\prime}, j, j^{\prime} \theta\right)}$, acting in $L^{2}\left(S_{1}\right)$, and given by

$$
\begin{aligned}
& \left(S_{(z, n)}^{\left(r, r^{\prime}, j^{\prime}, \theta\right)} f(\alpha)\right. \\
& \quad=e^{i n\left(\lambda+\lambda^{\prime}\right)} \theta^{j^{\prime} n} e^{-i n^{2}\left(j+j^{\prime}\right)} \alpha^{n\left(j+j^{\prime}\right)} e^{i\left(\left\langle r+r^{\prime} \theta\right) \alpha, z\right)} f\left(\alpha e^{-i n}\right) .
\end{aligned}
$$


Proposition 3.3. Each $S^{\left(r, r^{\prime}, j \cdot j^{\prime}, \theta\right)}$ is a multiplier representation whose multiplier is a coboundary. In fact, if $\tau(z, n)=e^{i n^{2}\left(\left(j+j^{\prime}\right) / 2\right)}$, then $\tau S^{\left(r, r^{\prime}, j, j^{\prime}, \theta\right)}$ is unitary.

Proof. Direct calculation of the multiplier.

We observe next that the unitary representations just constructed are special cases of the following.

DEFINITION 3.4. Let $r$ be a positive real number, $\lambda$ a real number, and $d$ an integer. Define a representation $V^{(r \cdot \lambda \cdot d)}$ of $D$, acting in $L^{2}\left(S_{1}\right)$, and given by

$$
\left(V_{(z, n)}^{(r, \lambda, d)} f\right)(\alpha)=e^{-i n^{2}}(d / 2) e^{i \lambda n} \alpha^{n d} e^{i(r \alpha, z)} f\left(\alpha e^{-i n}\right) .
$$

Proposition 3.5. Each of the unitary representations $\tau S^{\left(r, r^{\prime}, j, j^{\prime}, \theta\right)}$ is equivalent to one of the $\left[V^{(r, \lambda, d)}\right]$.

Proof. This is mostly a matter of renaming some of the variables. The unitary operator $A$ defined by $A f(\alpha)=f\left(\alpha\left(\left(r+r^{i} \bar{\theta}\right) /\left\|r+r^{\prime} \theta\right\|^{2}\right)\right)$ is needed as well. We omit the simple details.

REMARK. The last proposition is not exactly correct because of the one case in which $r+r^{\prime} \theta=0$. We simply ignore that one degenerate case.

THEOREM 3.6. (i) Each $V^{(r, \lambda, d)}$ is irreducible.

(ii) Each $V^{(r, \lambda, d)}$ is associated with the Lebesgue measure quasiorbit on the circle $\|\chi\|=r$ in $\hat{C}$.

(iii) $V^{(r, \lambda, d)}$ is equivalent to $V^{\left(r^{\prime}, \lambda^{\prime}, d^{\prime}\right)}$ if and only if $r=r^{\prime}, d=d^{\prime}$, and $\lambda=\lambda^{\prime}+p+2 \pi q$ for some integers $p$ and $q$.

(iv) $V^{(r, \lambda, 0)}$ is equivalent to $\rho^{(r, \lambda)}$. See $\S I$.

Proof. The usual proof shows the irreducibility, i.e., any operator which commutes with all the $V_{(z, 0)}^{(r, \lambda, d)}$ must be a multiplication operator, and any multiplication operator which commutes with all the $V_{(0, n)}^{(r, \lambda, d)}$ must be a constant. The restriction of $V^{(r, \lambda, d)}$ to the subgroup $C$ of $D$ is clearly concentrated on the Lebesgue measure quasi-orbit $\|\chi\|=$ $r$. Also it is immediate that $V^{(r, \lambda, 0)}$ is the same as $\rho^{(r, \lambda)}$. It remains only to prove (iii).

Suppose then that $V^{(r, \lambda, d)}$ is equivalent to $V^{\left(r^{\prime}, \lambda^{\prime}, d^{\prime}\right)}$. We may as well assume that $d \neq 0$ since when $d=0$ part (iii) follows from part (iv) and Theorem 1.5. By restricting to the subgroup $C$, we see that $r$ must be $r^{\prime}$. Next consider the restrictions of these representations 
to the subgroup $Z$ of $D$. We have that

$$
\left(V_{(0, n)}^{(r, \lambda), d)} f\right)(\alpha)=e^{-i n^{2}(d / 2)} e^{i \lambda n} \alpha^{n d} f\left(\alpha e^{-i n}\right) .
$$

For each integer $k$, for $0 \leqq k<|d|$, we have that the subspace $X_{k}$ of $L^{2}\left(S_{1}\right)$ spanned by the functions [ $\left.\alpha^{m d+k}\right], m$ running through the integers, is invariant. We may identify $X_{k}$ in the obvious way with the space $l^{2}$ and see that

$$
\left(\left.\left(\left.V^{(r, \lambda, d)}\right|_{z}\right)\right|_{\left(x_{k}\right)}\right)
$$

is equivalent to the representation $\pi$ of $Z$, acting in $l^{2}$, and given by $\left(\pi_{n} f\right)(m)=q(n, m) f(m+n)$, where $q$ is a function of modulus 1 . By the lemma below, any such representation $\pi$ is equivalent to the regular representation. Therefore $\left.V^{(r, \lambda, d)}\right|_{z}$ is equivalent to $|d|$ times the the regular representation of $Z$. It therefore follows that $|d|=$ $\left|d^{\prime}\right|$.

We must rule out the possibility that $d^{\prime}=-d$. By way of contradiction then suppose that $d^{\prime}$ is $-d$. Let $A$ be an operator which effects an equivalence between $V^{(r, \lambda, d)}$ and $\left.V^{\left(r, \lambda^{\prime},-d\right.}\right)$. Then $A$ actually commutes with all the operators $\left[V_{(z, 0)}^{(r, \lambda, d)}\right]$, (since these operators are the same as the operator $\left[V_{(z, 0)}^{\left(r, \lambda^{\prime},-d\right)}\right]$, and therefore $A$ is a multiplication operator $M$. We then obtain that

$$
\begin{gathered}
M(\alpha) \bar{M}\left(\alpha e^{-i n}\right) e^{-i n^{2}(d / 2)} e^{i \lambda n} \alpha^{n d} f\left(\alpha e^{-i n}\right) \\
=e^{i n^{2}(d / 2)} e^{i \lambda^{\prime} n} \alpha^{-n d} f\left(\alpha e^{-i n}\right)
\end{gathered}
$$

from which we obtain that

$$
\bar{M}\left(\alpha e^{-i n}\right)=\bar{M}(\alpha) e^{i d n^{2}} e^{i n\left(\lambda^{\prime}-\lambda\right)} \alpha^{-2 n d} .
$$

Expanding $M$ in its Fourier series, $M(\alpha)=\Sigma c_{k} \alpha^{k}$, and equating like coefficients, we have the following recursion relation:

$$
c_{k}=c_{k+n d} e^{-i d n^{2}} e^{-i n\left(\lambda^{\prime}-\lambda\right)},
$$

which would imply that the sequence $\left[c_{k}\right]$ is not square-summable unless it is identically zero. Since $A$ is unitary, $M$ cannot be zero. Hence we arrive at a contradiction and $d^{\prime}$ must be $d$.

To see the necessary relation between $\lambda$ and $\lambda^{\prime}$, again let $A$ be an operator effecting the equivalence. Then as before $A$ is a multiplication operator $M$. This time we have

$$
\bar{M}\left(\alpha e^{-i n}\right)=\bar{M}(\alpha) e^{i n\left(\lambda^{\prime}-\lambda\right)} .
$$

Comparing Fourier coefficients gives that $e^{i n\left(\lambda^{\prime}-\lambda\right)}=e^{i n p}$ for any $p$ such that $c_{p} \neq 0$. This shows that $\lambda=\lambda^{\prime}+p+2 \pi q$ for some integers $p$ and $q$ as desired. 
Finally, if $A$ is the operator on $L^{2}\left(S_{1}\right)$ defined by $A f(\alpha)=\alpha^{p} f(\alpha)$, then $A$ effects an equivalence between $V^{(r, \lambda, d)}$ and $V^{(r, \lambda+p, d)}$. Clearly adding a multiple of $2 \pi$ to $\lambda$ does not change the formula for $V^{(r, \lambda, d)}$ in any way. The theorem is then complete modulo the following lemma.

LEMMA 3.7. Let $\pi$ be a representation of the group $Z$, acting in $l^{2}$, and given by the formula $\left(\pi_{n} f\right)(m)=q(n, m) f(m+n)$, where $q$ is a scalar function of modulus 1 . Then $\pi$ is equivalent to the regular representation $L$ of $Z$.

Proof. Let $\delta$ be the element of $l^{2}$ with coordinates $\delta_{0}=1$ and $\delta_{m}=0$ for $m \neq 0$. If $\varphi_{n}=L_{n} \delta$, then $\left[\varphi_{n}\right]$ forms an orthonormal basis of $l^{2}$. If $\psi_{n}=\pi_{n} \delta$, then $\left[\psi_{n}\right]$ forms an orthonormal basis of $l^{2}$. Let $A$ be the unitary operator on $l^{2}$ which maps $\phi_{n}$ to $\psi_{n}$. Then:

$$
\begin{aligned}
\left(A L_{k} A^{-1}\right)\left(\psi_{n}\right) & =\left(A L_{k}\right)\left(\varphi_{n}\right)=A\left(L_{k} L_{n} \delta\right)=A\left(\varphi_{n+k}\right) \\
& =\psi_{n+k}=\pi_{k}\left(\psi_{n}\right),
\end{aligned}
$$

which proves that $A L_{k} A^{-1}=\pi_{k}$.

IV. Some new representations of the Mautner group.

D\&Finition 4.1. Let $r$ and $s$ be positive numbers, let $\lambda$ and $\mu$ be real numbers, and let $d$ be an integer. Define a mapping $U^{\langle r, s, \lambda, \mu, d)}$ of the Mautner group $G$ into the unitary operators on the Hilbert space $L^{2}\left(S_{1} \times S_{1}\right)$ by

$$
\begin{aligned}
& \left(U_{(z, w, i t)}^{(r, s, \lambda, \mu, d)} f\right)\left(\alpha, e^{2 \pi i \theta}\right) \\
& =e^{-i(d / 2)[1+t-\theta+[\theta]]^{2}} e^{i \lambda[1+t-\theta+[\theta]]} e^{i d \mu[1+t-\theta+[\theta]]} \\
& \quad \times \alpha^{d[1+t-\theta+[\theta]]} e^{i d(1-\theta+[\theta])[1+t-\theta+[\theta]]} \\
& \quad \times e^{i(r \alpha, z)} e^{i\left(s e^{2 \pi i \theta}, w\right)} f\left(\alpha e^{-i t}, e^{2 \pi i \theta} e^{-2 \pi i t}\right),
\end{aligned}
$$

where as usual (, ) denotes the real inner product in the complex plane, and where the square brackets denote the greatest integer function.

THEOREM 4.2. (i) Each $U^{(r, s, \lambda, \mu, d)}$ is an irreducible unitary representation of $G$.

(ii) Each $U^{(r, s, \lambda, \mu, d)}$ is associated with the Lebesgue measure quasi-orbit on the torus $S_{r} \times S_{s}$ with multiplicity 1 in $\left[C^{2}\right]^{\wedge}$.

(iii) For all values of $\mu$, we have that $U^{(r, s, \lambda, \mu, 0)}$ is equivalent to the representation $\pi^{(r, s, \lambda)}$ deformed in $\S I$.

(iv) Suppose $d \neq 0$. Then $U^{(r, s, \lambda, \mu, d)}$ is equivalent to $U^{\left(r^{\prime}, s^{\prime}, \lambda^{\prime}, \mu^{\prime}, d\right)}$ if and only if $r=r^{\prime}, s=s^{\prime}, d=d^{\prime}$, and $\lambda+d \mu=\lambda^{\prime}+d^{\prime} \mu^{\prime}+p+2 \pi q$ 
for some integers $p$ and $q$.

(v) If $d \neq 0$, then the representation $U^{(r, s, \lambda, \mu, d)}$ is new.

Proof. One can check, with tenacity, directly the $U^{(r, s, \lambda, \mu, d)}$ is multiplicative and irreducible. However we shall obtain this formula as a representation equivalent to an induced representation as in Theorem 1.4. Part (i) will follow from that. Part (ii) is clear simply by restricting this formula to the elements $(z, w, 0)$. Part (iii) is obvious, and part (v) certainly follows from part (iv) and remarks in $\S I$.

We let $N$ be the closed normal subgroup of $G$ consisting of the triples $(0, w, 0)$, and let $\varphi$ be the character of $N$ defined by the complex number $\varphi=s e^{2 \pi i \mu}$. Recall that the "Little Group" for $\phi$ is the discrete Mautner group $D$, and take $V$ to be the representation $V^{(r, \lambda, d)}$ of $D$. Define the representation $S$ on the stability subgroup $H$ of $G$ for $\varphi$ by $S_{(z, w, n)}=e^{i \varphi,(w)} V_{(z, n)}$. Let $U$ be the induced representation $\operatorname{ind}_{H}^{G} S$, and let us show that $U$ is equivalent to $U^{(r, s, \lambda, \mu, d)}$.

The mapping $(z, w, t) \rightarrow e^{2 \pi i t}$ maps $G$ onto the coset space $G / H$, which is equivalent to $S_{1}$. We take for our cross-section of $S_{1}$ into $G$, see [2], the mapping $\gamma$ defined by $\gamma\left(e^{2 \pi i \theta}\right)=(0,0, \theta-[\theta])$. The space of $U$ then is the tensor product of $L^{2}\left(S_{1}\right)$ with the Hilbert space of the representation $S$, i.e., the space of $U$ is $L^{2}\left(S_{1}\right) \otimes L^{2}\left(S_{1}\right)$. If $g$ and $f$ both belong to $L^{2}\left(S_{1}\right)$, then the formula for $U$ is given by

$$
\begin{aligned}
& \left(\left(U_{(z, w, t)}(g \otimes f)\right) \mid g \otimes f\right) \\
& =\int_{S_{1}} g\left(s_{1} \cdot(z, w, t)\right) \bar{g}\left(s_{1}\right)\left(\left(S_{\left(\gamma\left(s_{1}\right)(z, w, t)\left(\gamma\left(s_{1}(z, w, t)\right)\right)-1\right)} f\right) \mid f\right) d s_{1} \\
& =\int_{[0,1)} g\left(e^{2 \pi i(\theta+t)}\right) \bar{g}\left(e^{2 \pi i \theta}\right)\left(\left(S_{((0,0, \theta)(z, w, t)(0,0, \theta+t-[\theta+t])-1)} f\right) \mid f\right) d \theta
\end{aligned}
$$

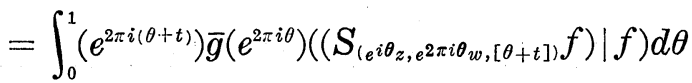

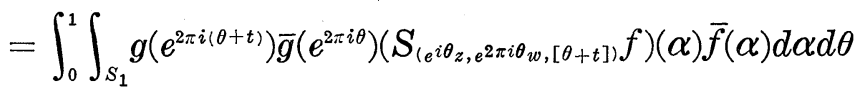

$$
\begin{aligned}
& =\int_{0}^{1} \int_{S_{1}} g\left(e^{2 \pi i(\theta+t)}\right) \bar{g}\left(e^{2 \pi i \theta)}\right) e^{i(\varphi, e 2 \pi i \theta w)} e^{-i(d / 2)[\theta+t]^{2}} e^{i \lambda[\theta+t]} \alpha^{d[\theta+t]} \\
& \times e^{i\left(r \alpha, e^{\left.i \theta_{z}\right)}\right.} f\left(\alpha e^{-i[\theta+t]}\right) \bar{f}(\alpha) d \alpha d \theta \text {. }
\end{aligned}
$$

From this formula we can derive the action of $U$ acting in $L^{2}\left(S_{1} \times S_{1}\right)$. Thus

$$
\begin{aligned}
& \left(U_{(z, w, t)} f\right)\left(\alpha, e^{2 \pi i \theta}\right) \\
& =e^{-i(d / 2)[\theta-[\theta]+t]^{2}} e^{i \lambda[\theta-[\theta]+t]} \alpha^{d[\theta-[\theta]+t]} \\
& \times e^{i\left(r e^{-i(\theta-[\theta]) \alpha, z)}\right.} e^{i\left(s e 2 \pi i \mu_{e}-2 \pi i(\theta-[\theta]), w\right)} f\left(\alpha e^{-i[\theta-[\theta]+t]}, e^{2 \pi i \theta} e^{2 \pi i t}\right) .
\end{aligned}
$$

Now we conjugate by the operator $A$ defined on $L^{2}\left(S_{1} \times S_{1}\right)$ by 
$A f\left(\alpha, e^{2 \pi i \theta}\right)=f\left(\alpha, e^{-2 \pi i \theta}\right)$, and then by the operator $B$ defined by $\left.B f\left(\alpha, e^{2 \pi i \theta}\right)=f\left(\alpha e^{i(1-\theta+[\theta]}\right), e^{2 \pi i \theta}\right)$. This converts $U$ into an equivalent representation $U^{\prime}$ of the form

$$
\begin{aligned}
\left(U_{(z, w, t)}^{\prime}\right. & f)\left(\alpha, e^{2 \pi i \theta}\right) \\
= & e^{-i(d / 2)[1+t-\theta+[\theta]]^{2}} e^{i \lambda[1+t-\theta+[\theta]]} \alpha^{d[1+t-\theta+[\theta]]} \\
& \times e^{i d(1-\theta+[\theta])[1+t-\theta+[\theta]]} e^{i(r \alpha, z)} \\
& \times e^{i\left(s e 2 \pi i \mu_{e} 2 \pi i \theta, w\right)} f\left(\alpha e^{-i t}, e^{2 \pi i \theta} e^{-2 \pi i t}\right) .
\end{aligned}
$$

Conjugating next by the operator $U_{(0,0, \mu)}^{\prime}$ and then by the operator $C$ defined by $C f\left(\alpha, e^{2 \pi i \theta}\right)=f\left(\alpha e^{i \mu}, e^{2 \pi i \theta}\right)$, we see that $U$ is equivalent to $U^{(r, s, \lambda, \mu, d)}$. This verifies part (i) of the theorem. More to the point, it indicates where Definition 4.1 comes from.

Now to the proof of part (iv). Suppose that $U^{(r, s, \lambda, \mu, d)}$ is equivalent to $U^{\left(r^{\prime}, s^{\prime}, \lambda^{\prime}, \mu^{\prime}, d^{\prime}\right)}$. Then by restricting to the subgroup $C^{2}$ we see that we must have $r=r^{\prime}$ and $s=s^{\prime}$. Further, if $A$ is an operator effecting the equivalence between these representations, then as usual it must be a multiplication operator $M$. Setting $z=w=0$, we obtain

$$
\begin{aligned}
M(\alpha, & \left.e^{2 \pi i \theta}\right) \bar{M}\left(\alpha e^{-i t}, e^{2 \pi i(\theta-t)}\right) e^{-i(d / 2)[1+t-\theta+[\theta]] 2} \\
& \times e^{i \lambda[1+t-\theta+[\theta]]} e^{i d \mu[1+t-\theta+[\theta]]} \alpha^{d[1+t-\theta+[\theta]]} e^{i d(1-\theta+[\theta])[1+t-\theta+[\theta]]} \\
= & e^{-i\left(d^{\prime} / 2\right)[1+t-\theta+[\theta]] 2} e^{i \lambda^{\prime}[1+t-\theta+[\theta]]} e^{i d^{\prime} \mu^{\prime}[1+t-\theta+[\theta]]} \\
& \times \alpha^{d^{\prime}[1+t-\theta+[\theta]]} e^{i d^{\prime}(1-\theta+[\theta])[1+t-\theta+[\theta]]},
\end{aligned}
$$

from which it follows that

$$
\begin{aligned}
& \bar{M}\left(\alpha e^{-i t}, e^{2 \pi i(\theta-t)}\right) \\
& \quad=c(t, \theta) \bar{M}\left(\alpha, e^{2 \pi i \theta}\right) \alpha^{\left(d^{\prime}-d\right)[1+t-\theta+[\theta]]},
\end{aligned}
$$

where $c(t, \theta)$ is a function of modulus 1 . We may now conclude that $d=d^{\prime}$ by expanding $M$ in its Fourier series in $\alpha$ for fixed $\theta$, then fixing $t$ as well, and then arguing just as in the proof to Theorem 3.6.

Knowing that $d=d^{\prime}$ simplifies the above equation into the form

$$
\begin{aligned}
& \bar{M}\left(\alpha e^{-i t}, e^{2 \pi i(\theta-t)}\right) \\
& \quad=\bar{M}\left(\alpha, e^{2 \pi i \theta}\right) e^{i\left(\left(\lambda^{\prime}+d \mu^{\prime}\right)-(\lambda+d \mu)\right)[1+t-\theta+[\theta]]} .
\end{aligned}
$$

Writing $M$ in its Fourier series as $M\left(\alpha, e^{2 \pi i \theta}\right)=\Sigma \Sigma c_{j k} \alpha^{j} e^{2 \pi i k \theta}$, setting $t=1$, and comparing like coefficients, gives us that $e^{i j}=e^{i\left(\left(\lambda^{\prime}+d \mu^{\prime}\right)-(\lambda+d \mu),\right.}$ for every $j$ such that $c_{j k} \neq 0$ for some $k$. Since some such $j$ must exist, it follows that $\left(\lambda^{\prime}+d \mu^{\prime}\right)-(\lambda+d \mu)=p+2 \pi q$ for some integers $p$ and $q$. This proves half of (iv).

Finally suppose that $r=r^{\prime}, s=s^{\prime}, d=d^{\prime}$, and that $\lambda+d \mu=$ $\lambda^{\prime}+d \mu^{\prime}+p+2 \pi q$ for some integers $p$ and $q$. Set $\lambda^{\prime \prime}=\lambda^{\prime}-p-2 \pi q$. 
Then by Theorem 3.6 we have that the representations $V^{\left(r, \lambda^{\prime}, d\right)}$ and $V^{\left(r, \lambda^{\prime \prime}, d\right)}$ are equivalent, and therefore by Theorem 1.4 the representions $U^{\left(r, s, \lambda^{\prime}, \mu^{\prime}, d\right)}$ and $U^{\left(r, s, \lambda^{\prime \prime}, \mu^{\prime}, d\right)}$ are equivalent. (The same character $\varphi=$ $s e^{2 \pi i \mu^{\prime}}$ is being used in both cases.) Hence we need only verify that $U^{(r, s, \lambda, \mu, d)}$ is equivalent to $U^{\left(r, s, \lambda^{\prime \prime}, \mu^{\prime}, d\right)}$. But this is immediate from the definitions since $\lambda^{\prime \prime}+d \mu^{\prime}=\lambda+d \mu$. This completes the proof.

REMARK 1. Because $U^{(r, s, \lambda, \mu, d)}$ is equivalent to $U^{\left(r, s, \lambda+d\left(\mu-\mu^{\prime}\right), \mu^{\prime}, d\right)}$, it is clear that for each fixed $\mu$ the same set of representations of $G$ occurs in this series, but with a slightly different parametrization. In other words the set $\left[U^{(r, s, \lambda, \mu, d)}\right]$ of representations of $G$ depends only on the orbit of the character $s e^{2 \pi i \mu}$. This is in analogy with Mackey's procedure.

REMARK 2. The equivalence relation among parameters is one of the famous "Bad" equivalence relations. This was to be expected since the dual was known to be nonsmooth.

REMARK 3. All the representations $U^{(r . s, \lambda, \mu, d)}$ have multiplicity 1 on $C^{2}$. The author has tried without success to decide whether these representations exhaust all such representations of $G$.

REMARK 4. Beginning with the work of Auslander and Moore, and proceeding through the research of many other mathematicians, Kenstant, Pukanski, Kirillov, etc.), the unitary representation theory for solvable group has been extensively investigated. Much of this work has been directed toward the type I case, so that the results are not applicable to the particular solvable group we have been studying. For nontype I groups, most researchers have abandoned the investigation of individual irreducible unitary representations and have chosen to pursue "more tractible" problems, e.g., the decomposition of a reducible representation into primary constituents, and the determination of the Plancherel formula. To the author's knowledge, no other techniques have led to a "New" irreducible representations of the Mautner group, or for that matter to any nontype I connected solvable Lie group. The fact that our methods have succeeded in some small way we take then as an encouraging sign that the individual irreducible representation can be investigated, despite the lack of smoothness in the dual.

\section{REFERENCES}

1. L. Baggett, Multiplier extensions other than the Mackey extension, PAMS, 56 (1976), 351-356. 
2. G. Mackey, Induced representations of locally compact groups I, Annals Math., 55 (1952), 101-139.

3. — Unitary representations of group extensions I, Acta Math., 99 (1958), 265-311.

4. F. I. Mautner, Unitary representations of locally compact groups II, Annals Math., 5 (1950), 528-556.

5. A. Ramsay, Nontransitive quasi-orbits in Mackey's analysis of group extensions, Acta Math., 137 (1976), 17-48.

Received October 28, 1977.

UNIVERSITY OF COLORADO

BOULDER, CO 80309 


\section{PACIFIC JOURNAL OF MATHEMATICS}

\section{EDITORS}

RICHARD ARENS (Managing Editor)

University of California

Los Angeles, California 90024

C. W. Curtis

University of Oregon

Eugene, OR 97403

C. C. MOORE

University of California

Berkeley, CA 94720

\section{J. DUGUNDJI}

Department of Mathematics University of Southern California Los Angeles, California 90007

R. Finn AND J. Milgram Stanford University Stanford, California 94305

\section{ASSOCIATE EDITORS}

E. F. BeCK ENBACH

B. H. NeUMaNN

F. WOLF

K. Yoshida

\section{SUPPORTING INSTITUTIONS}

UNIVERSITY OF BRITISH COLUMBIA CALIFORNIA INSTITUTE OF TECHNOLOGY UNIVERSITY OF CALIFORNIA MONTANA STATE UNIVERSITY UNIVERSITY OF NEVADA, RENO NEW MEXICO STATE UNIVERSITY OREGON STATE UNIVERSITY UNIVERSITY OF OREGON
UNIVERSITY OF SOUTHERN CALIFORNIA STANFORD UNIVERSITY UNIVERSITY OF HAWAII UNIVERSITY OF TOKYO UNIVERSITY OF UTAH WASHINGTON STATE UNIVERSITY UNIVERSITY OF WASHINGTON 


\section{Pacific Journal of Mathematics \\ Vol. 77, No. $1 \quad$ January, 1978}

Dan Amir, Chebyshev centers and uniform convexity ............... 1

Lawrence Wasson Baggett, Representations of the Mautner group. I ..... 7

George Benke, Trigonometric approximation theory in compact totally

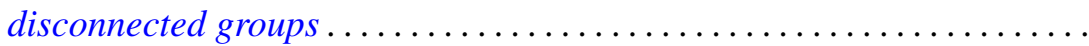

M. Bianchini, O. W. Paques and M. C. Zaine, On the strong compact-ported topology for spaces of holomorphic mappings ..................

Marilyn Breen, Sets with $(d-2)$-dimensional kernels

J. L. Brenner and Allen Kenneth Charnow, Free semigroups of $2 \times 2$

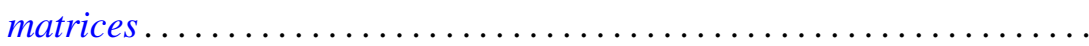

David Bressoud, A new family of partition identities .................

David Fleming Dawson, Summability of matrix transforms of stretchings

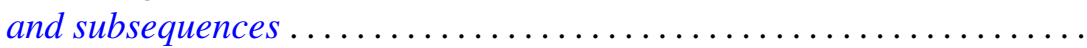

Harold George Diamond and Paul Erdôs, A measure of the nonmonotonicity

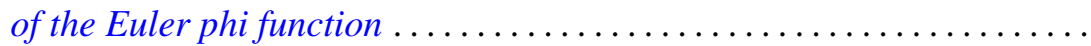

Gary Doyle Faulkner and Ronald Wesley Shonkwiler, Kernel dilation in reproducing kernel Hilbert space and its application to moment

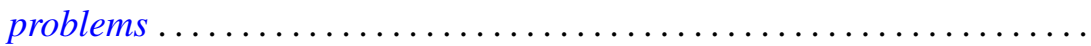

Jan Maksymilian Gronski, Classification of closed sets of attainability in the

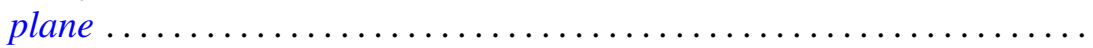

H. B. Hamilton and T. E. Nordahl, Semigroups whose lattice of congruences

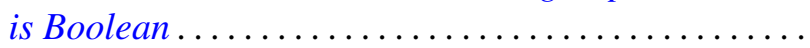

Harvey Bayard Keynes and D. Newton, Minimal $(G, \tau)$-extensions ...

Anthony To-Ming Lau, The Fourier-Stieltjes algebra of a topological

semigroup with involution.

B. C. Oltikar and Luis Ribes, On prosupersolvable groups ...

Brian Lee Peterson, Extensions of pro-affine algebraic groups ...

Thomas M. Phillips, Primitive extensions of Aronszajn spaces ...

Mehdi Radjabalipour, Equivalence of decomposable and 2-decomposable operators. .

M. Satyanarayana, Naturally totally ordered semigroups .

Fred Rex Sinal, A homeomorphism classification of wildly imbedded two-spheres in $S^{3}$

Hugh C. Williams, Some properties of a special set of recurring

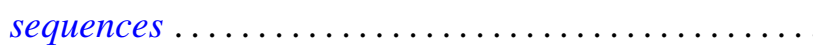

